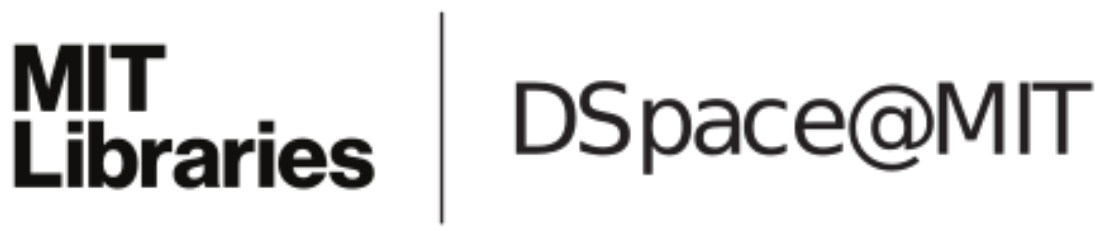

\author{
MIT Open Access Articles
}

\begin{abstract}
Broken translational symmetry in an emergent paramagnetic phase of graphene
\end{abstract}

The MIT Faculty has made this article openly available. Please share how this access benefits you. Your story matters.

Citation: Chern, Gia-Wei et al. "Broken Translational Symmetry in an Emergent Paramagnetic Phase of Graphene." Physical Review B 86.11 (2012). (C2012 American Physical Society

As Published: http://dx.doi.org/10.1103/PhysRevB.86.115443

Publisher: American Physical Society

Persistent URL: http://hdl.handle.net/1721.1/75757

Version: Final published version: final published article, as it appeared in a journal, conference proceedings, or other formally published context

Terms of Use: Article is made available in accordance with the publisher's policy and may be subject to US copyright law. Please refer to the publisher's site for terms of use. 


\title{
Broken translational symmetry in an emergent paramagnetic phase of graphene
}

\author{
Gia-Wei Chern, ${ }^{1,2}$ Rafael M. Fernandes, ${ }^{3,2}$ Rahul Nandkishore, ${ }^{4,5}$ and Andrey V. Chubukov ${ }^{1}$ \\ ${ }^{1}$ Department of Physics, University of Wisconsin-Madison, Madison, Wisconsin 53706, USA \\ ${ }^{2}$ Theoretical Division, Los Alamos National Laboratory, Los Alamos, New Mexico 87545, USA \\ ${ }^{3}$ Department of Physics, Columbia University, New York, New York 10027, USA \\ ${ }^{4}$ Department of Physics, Massachusetts Institute of Technology, Cambridge, Massachusetts 02139, USA \\ ${ }^{5}$ Princeton Center for Theoretical Science, Princeton University, Princeton, NJ 08540, USA
}

(Received 27 March 2012; published 26 September 2012)

\begin{abstract}
We show that the spin-density wave state on the partially filled honeycomb and triangular lattices is preempted by a paramagnetic phase that breaks an emergent $Z_{4}$ symmetry of the system associated with the four inequivalent arrangements of spins in the quadrupled unit cell. Unlike other emergent paramagnetic phases in itinerant and localized-spin systems, this state preserves the $C_{6}$ rotational symmetry of the lattice, but breaks its translational symmetry, giving rise to a superlattice structure that can be detected by scanning tunneling microscopy. This emergent phase also has distinctive signatures in the magnetic spectrum that can be probed experimentally.
\end{abstract}

DOI: 10.1103/PhysRevB.86.115443

PACS number(s): 73.22.Pr, 73.22.Gk, 75.25.Dk

\section{INTRODUCTION}

Unconventional paramagnetic phases are characterized not only by the absence of long-range spin order, but also by a broken symmetry related to new degrees of freedom that emerge from the collective magnetic behavior of the system. As a result, their elementary excitations and thermodynamic properties are rather different than those of an ordinary paramagnet. These phases usually appear in frustrated systems with localized spins, as a result of the interplay between frustration and fluctuations. Canonical examples include the Ising-nematic phase of the extended Heisenberg model on the square lattice, ${ }^{1}$ the spin-nematic phase of the Heisenberg model on the kagome lattice, ${ }^{2}$ and the magnetic-charge ordered phase in kagome spin ice. ${ }^{3}$ Itinerant magnetic systems can also display paramagnetic phases with unusual broken symmetries. This is believed to be the case in the ruthenates ${ }^{4}$ and in the iron-based superconductors. ${ }^{5-7}$ In these systems, the emergent paramagnetic phase breaks the lattice rotational symmetry, while the spin-rotational and lattice translational symmetries remain preserved.

In this paper, we present an unusual itinerant paramagnetic phase that breaks the translational invariance without changing the point-group symmetry of the lattice. This phase arises in partially filled hexagonal (triangular and honeycomb) lattices, preempting a spin-density wave (SDW) order, and could potentially be realized in single-layer graphene doped near the saddle point of the band structure ( $3 / 8$ or $5 / 8$ filling) ${ }^{8,9}$ The SDW order below $T_{N}$ for fermions on a hexagonal lattice is uniaxial, with all spins pointing along the same direction. ${ }^{10}$ The magnetic unit cell contains eight sites, six of which have moment $-\boldsymbol{\Delta}$ and two have moment $3 \boldsymbol{\Delta}$ (see Fig. 1). This state breaks not only the $O(3)$ spin-rotational symmetry, but also a discrete $Z_{4}$ symmetry related to the four inequivalent choices for the positions of the large $3 \Delta$ spin moments in the eight-site unit cell. These four inequivalent spin configurations transform into each other upon translation of the origin of coordinates to neighboring hexagons, from point $\mathrm{A}$ to points $\mathrm{B}, \mathrm{C}$, and $\mathrm{D}$ in Fig. 1 . Thus, breaking the $Z_{4}$ symmetry corresponds to breaking the translational symmetry of the lattice.

Of course, once the $O(3)$ symmetry is broken, the $Z_{4}$ symmetry has to be broken too. We show, however, that the $Z_{4}$ symmetry breaks down at higher temperatures than the $O(3)$ symmetry. As a result, the SDW ordering at $T_{N}$ is preempted by a phase transition at $T_{Z_{4}}>T_{N}$, which falls into the universality class of the four-state Potts model. In the $Z_{4}$ phase at $T_{N}<T<T_{Z_{4}},\left\langle\mathbf{S}_{i}\right\rangle=0$ for all sites (i.e., this phase is a paramagnet), and the unit cell consists of the highlighted sites in Fig. 2. The $C_{6}$ rotational symmetry of the lattice is preserved. Yet, the unit cell has eight inequivalent sites: for six of them $(i=1, \ldots, 6$ in Fig. 2$)$ the bond correlators $\left\langle\mathbf{S}_{i} \cdot \mathbf{S}_{i+\delta}\right\rangle$ with their nearest neighbors are $\Delta^{2}$ and $-3 \Delta^{2}$ [blue and red bonds in Fig. 1(b)], while for the remaining two sites $(i=7,8)$ all bond correlators are $-3 \Delta^{2}$. The broken $Z_{4}$ symmetry corresponds to choosing these two "special" sites out of the eight sites in the unit cell. One such choice is shown in Fig. 2. One can easily verify that the other three choices correspond to moving the origin of the coordinates from $\mathrm{A}$ to one of the points B, C, or D in Fig. 2. This obviously implies that the translational symmetry of the lattice is broken. Experimentally, the quadrupled unit cell in the $Z_{4}$ phase can be readily probed by scanning tunneling microscopy (STM). Furthermore, we show that the transition to this phase is accompanied by a jump of the staggered spin susceptibility, which can be probed by neutron scattering or nuclear magnetic resonance (NMR).

\section{UNIAXIAL SDW ORDER}

The Fermi surface (FS) of graphene near $3 / 8$ or $5 / 8$ filling is near-nested and contains three saddle points with nearly vanishing Fermi velocity [the three $M_{a}$ points in Fig. 3(a)]. Pairs of inequivalent $M_{a}$ points are connected by three commensurate nesting vectors $\mathbf{Q}_{1}=(0,2 \pi / \sqrt{3})$ and $\mathbf{Q}_{2,3}=(\mp \pi,-\pi / \sqrt{3})$. The divergent density of states at the $M$ points makes doped graphene a fertile ground for exploring nontrivial many-body density-wave and superconducting states. ${ }^{11-19}$ The SDW instability is subleading to a chiral $d$-wave superconductivity exactly at $3 / 8$ or $5 / 8$ filling, ${ }^{11}$ but can become the leading instability slightly away from $3 / 8$ or $5 / 8$ filling. ${ }^{10,16}$

In particular, the FS at the saddle-point doping levels, e.g., $3 / 8$ or $5 / 8$ for the honeycomb lattice, is a perfect hexagon inscribed within a hexagonal Brillouin zone (BZ) as shown in 

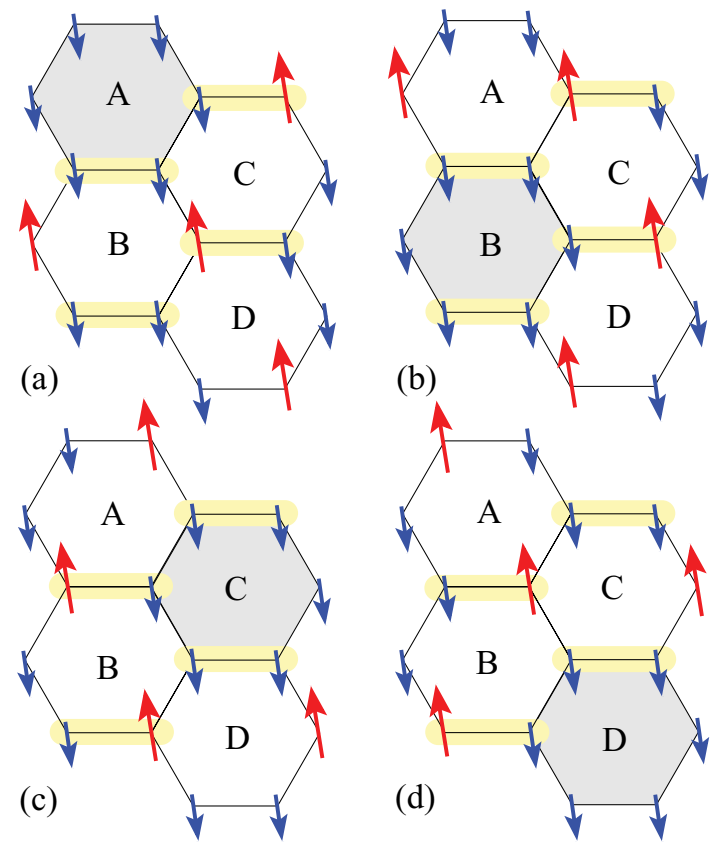

FIG. 1. (Color online) The four inequivalent uniaxial SDW states. The quadrupled unit cell is highlighted with yellow boxes. Among the eight sites of the enlarged unit cell, two have large spin moment $\left\langle\mathbf{S}_{i}\right\rangle=$ $3 \boldsymbol{\Delta}$ (red arrows) and six have small moment $\left\langle\mathbf{S}_{i}\right\rangle=-\boldsymbol{\Delta}$ (blue arrows). The total spin in each unit cell is zero. The four different states correspond to the additional $Z_{4}$ symmetry of the order-parameter manifold.

Fig. 3(a). This FS is completely nested by three wave vectors $\mathbf{Q}_{1}=(0,2 \pi / \sqrt{3})$, and $\mathbf{Q}_{2,3}=( \pm \pi,-\pi / \sqrt{3})$, and the nesting opens the door to a SDW instability. However, not all points on the Fermi surface are of equal importance. In particular, the three saddle points $M_{a}(a=1,2,3)$ give rise to a logarithmic singularity in the density of states (DOS) and control the SDW instability at weak coupling.

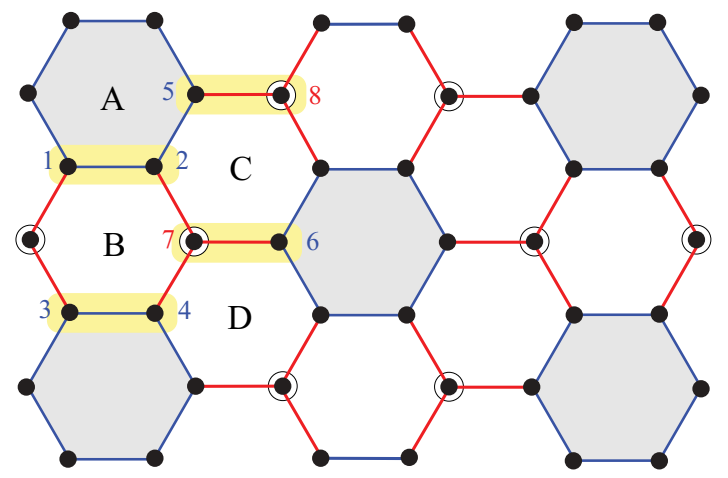

FIG. 2. (Color online) Schematic representation of the emergent $Z_{4}$ symmetry-breaking phase. The quadrupled unit cell is highlighted with yellow boxes; the numbers indicate the eight inequivalent sites. This state has the same broken translational symmetry as that of the SDW in Fig. 1(a), but $O(3)$ symmetry is preserved (no arrows). The nearest-neighbor correlation function $\left\langle\mathbf{S}_{i} \cdot \mathbf{S}_{j}\right\rangle$ is $\Delta^{2}$ for blue bonds and $-3 \Delta^{2}$ for red bonds. Other three states are obtained by moving the origin of coordinates from $\mathrm{A}$ to either $\mathrm{B}$ or $\mathrm{C}$ or $\mathrm{D}$.
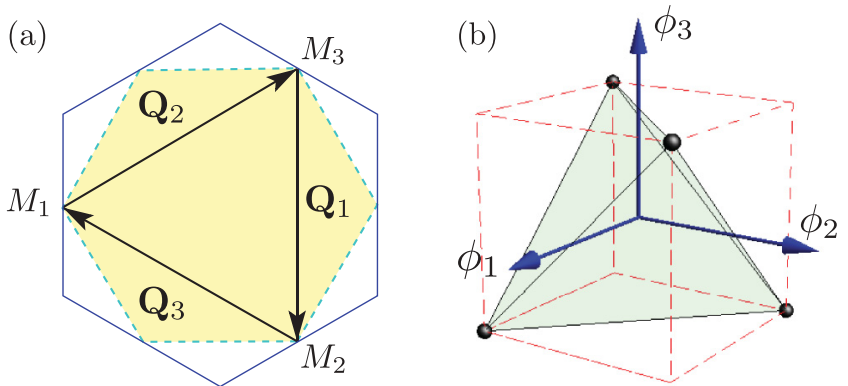

FIG. 3. (Color online) (a) The Fermi surface at the doping level of interest is a hexagon inscribed within the hexagonal Brillouin zone (BZ), for both honeycomb and triangular lattices. The FS has three saddle points $M_{a}$ located at the corners of the hexagon. Pairs of inequivalent saddle points are connected by three inequivalent nesting vectors $\mathbf{Q}_{1}=(0,2 \pi / \sqrt{3})$ and $\mathbf{Q}_{2,3}=(\mp \pi,-\pi / \sqrt{3})$. (b) Order-parameter space of the preemptive $Z_{4}$ phase. In the ordered phase, the vector order parameter $\phi=\left(\phi_{1}, \phi_{2}, \phi_{3}\right)$ points toward one of the four corners of a regular tetrahedron.

Thus, we consider the following Hamiltonian:

$$
\begin{aligned}
H= & \sum_{a=1,2,3} \varepsilon_{a} c_{a, \alpha}^{\dagger} c_{a, \alpha} \\
& -\sum_{a \neq b}\left(g_{2} c_{a, \alpha}^{\dagger} c_{b, \beta}^{\dagger} c_{b, \beta} c_{a, \alpha}+g_{3} c_{a, \alpha}^{\dagger} c_{a, \beta}^{\dagger} c_{b, \beta} c_{b, \alpha}\right),
\end{aligned}
$$

where $c_{a, \alpha}^{\dagger}$ creates electrons with spin $\alpha$ around the saddle point $M_{a}$. There are two electron-electron interactions that contribute to the SDW channel, namely, $g_{2}$ and $g_{3}$, which represent the forward and umklapp scatterings, respectively. The dispersions in the vicinity of the saddle points are

$$
\begin{gathered}
\varepsilon_{1}(\mathbf{k})=\frac{3 t_{1}}{4}\left(k_{y}^{2}-3 k_{x}^{2}\right), \\
\varepsilon_{2,3}(\mathbf{k})=-\frac{3 t_{1}}{4} 2 k_{y}\left(k_{y} \mp \sqrt{3} k_{x}\right),
\end{gathered}
$$

where $t_{1}$ is the nearest-neighbor hopping constant. The quartic interaction terms in Eq. (2) can be decoupled via the Hubbard-Stratonovich transformation with the SDW order parameters: $\boldsymbol{\Delta}_{i}=\boldsymbol{\Delta}_{a, b}=\frac{g_{2}+g_{3}}{3} \sum_{\mathbf{k}}\left\langle c_{a, \alpha}^{\dagger} \boldsymbol{\sigma}_{\alpha \beta} c_{b, \beta}\right\rangle$. Each of these vector order parameters corresponds to a nesting vector which connects two saddle points: $\mathbf{Q}_{i}=\mathbf{M}_{a}-\mathbf{M}_{b}$. The partition function of the system can then be written as $Z=\int \mathcal{D} c^{\dagger} \mathcal{D} c \mathcal{D} \boldsymbol{\Delta}_{i} \exp \left(-S\left[c^{\dagger}, c, \boldsymbol{\Delta}\right]\right)$ with

$$
\begin{aligned}
S\left[c, c^{\dagger}, \boldsymbol{\Delta}_{i}\right]= & \sum_{a} \int_{\tau} c_{a, \alpha}^{\dagger}\left(\partial_{\tau}-\varepsilon_{a}\right) c_{a, \alpha} \\
& +\frac{2}{g_{2}+g_{3}} \sum_{i} \int_{x, \tau}\left|\boldsymbol{\Delta}_{i}\right|^{2} \\
& -\sum_{a \neq b} \int_{\tau} \boldsymbol{\Delta}_{a, b} \cdot c_{a, \alpha}^{\dagger} \boldsymbol{\sigma}_{\alpha \beta} c_{b, \beta},
\end{aligned}
$$

where $\int_{\tau}=\int_{0}^{1 / T} d \tau$. The fermionic part becomes quadratic and can be integrated out. By expanding the resulting action 
to fourth order in $\boldsymbol{\Delta}_{i}$, we obtain the effective action

$$
\begin{aligned}
S\left[\boldsymbol{\Delta}_{i}\right] & r_{0} \sum_{i} \int_{x}\left|\boldsymbol{\Delta}_{i}\right|^{2}+\frac{u}{2} \int_{x}\left(\left|\boldsymbol{\Delta}_{1}\right|^{2}+\left|\boldsymbol{\Delta}_{2}\right|^{2}+\left|\boldsymbol{\Delta}_{3}\right|^{2}\right)^{2} \\
& +\frac{v}{2} \int_{x}\left[\left(\left|\boldsymbol{\Delta}_{1}\right|^{2}+\left|\boldsymbol{\Delta}_{2}\right|^{2}-2\left|\boldsymbol{\Delta}_{3}\right|^{2}\right)^{2}+3\left(\left|\boldsymbol{\Delta}_{1}\right|^{2}-\left|\boldsymbol{\Delta}_{2}\right|^{2}\right)^{2}\right] \\
& -\frac{g}{2} \int_{x}\left[\left(\boldsymbol{\Delta}_{1} \cdot \boldsymbol{\Delta}_{2}\right)^{2}+\left(\boldsymbol{\Delta}_{2} \cdot \boldsymbol{\Delta}_{3}\right)^{2}+\left(\boldsymbol{\Delta}_{3} \cdot \boldsymbol{\Delta}_{1}\right)^{2}\right]+\cdots .
\end{aligned}
$$

Here, $r_{0} \propto\left(T-T_{N}\right)$, where $T_{N}$ is the mean-field SDW transition temperature. The coefficients $u, v, g$ in Eq. (5) were calculated in Ref. 10 and found to be positive, with $v / u=1 / \ln \left(W / T_{N}\right) \ll 1$ and $g / u=\left(T_{N} / W\right) / \ln \left(W / T_{N}\right) \ll$ 1 , where $W$ is the bandwidth.

Minimizing $S\left[\boldsymbol{\Delta}_{i}\right]$ with respect to $\boldsymbol{\Delta}_{i}$ and neglecting momentarily the fluctuations of the $\boldsymbol{\Delta}_{i}$ fields, we see that $v>0$ implies that the magnitudes of $\boldsymbol{\Delta}_{i}$ are equal, while $g>0$ makes all $\boldsymbol{\Delta}_{i}$ collinear. The particular uniaxial state with $\left(\boldsymbol{\Delta}_{1}, \boldsymbol{\Delta}_{2}, \boldsymbol{\Delta}_{3}\right)=(\Delta, \Delta, \Delta) \hat{\mathbf{n}}$ is shown in Fig. 1(a). There exist, however, three other states with the same energy: $(\Delta,-\Delta,-\Delta) \hat{\mathbf{n}},(-\Delta, \Delta,-\Delta) \hat{\mathbf{n}}$, and $(-\Delta,-\Delta, \Delta) \hat{\mathbf{n}}$. These states can not be obtained from the one shown in Fig. 1(a) by a global spin rotation. Instead, these four degenerate states are related by a translational $Z_{4}$ symmetry: they transform into each other by moving the origin of coordinates from $A$ to $B$, C, or D [Figs. 1(b)-1(d)]. The ground state in Fig. 1(a) chooses a particular direction of $\hat{\mathbf{n}}$ and also one of the four positions of the origin of coordinates and therefore breaks $O(3) \times Z_{4}$ symmetry.

\section{PREEMPTIVE $Z_{4}$ PHASE}

\section{A. Order parameters}

We now allow $\Delta_{i}$ to fluctuate and analyze the possible emergence of a phase in which $Z_{4}$ symmetry is broken but $O$ (3) symmetry is preserved. In such a phase $\left\langle\boldsymbol{\Delta}_{i}\right\rangle=0$, but $\left\langle\boldsymbol{\Delta}_{i} \cdot \boldsymbol{\Delta}_{j}\right\rangle \neq 0$. A proper order parameter for the $Z_{4}$ phase is the triplet $\boldsymbol{\phi}=\left(\phi_{1}, \phi_{2}, \phi_{3}\right)$, where $\phi_{i}=g \boldsymbol{\Delta}_{j} \cdot \boldsymbol{\Delta}_{k}$ and $(i j k)$ are cyclic permutations of (123). The $Z_{4}$ symmetry-breaking phase has $\left\langle\phi_{i}\right\rangle= \pm \phi$, with the constraint $\phi_{1} \phi_{2} \phi_{3}>0$. To investigate whether this state emerges, we go beyond the mean-field approximation for $S\left[\boldsymbol{\Delta}_{i}\right]$ by including fluctuations of the $\boldsymbol{\Delta}_{i}$ fields, and reexpress the action in terms of the collective variables $\phi_{i}$. We analyze this action assuming that fluctuations of $\phi_{i}$ are weak and check whether a nonzero $\left\langle\phi_{i}\right\rangle$ emerges above the SDW transition temperature.

To obtain the action in terms of $\phi_{i}$, we apply a HubbardStratonovich transformation ${ }^{6}$ and introduce six auxiliary fields, one for each quartic term. These six fields include two fields $\zeta_{1} \propto\left(\boldsymbol{\Delta}_{1}^{2}+\boldsymbol{\Delta}_{2}^{2}-2 \boldsymbol{\Delta}_{2}^{2}\right)$ and $\zeta_{2} \propto\left(\boldsymbol{\Delta}_{1}^{2}-\boldsymbol{\Delta}_{2}^{2}\right)$ which break the $C_{6}$ rotational symmetry, the three fields $\phi_{i} \propto \boldsymbol{\Delta}_{j} \cdot \boldsymbol{\Delta}_{k}$ associated with the $Z_{4}$ symmetry breaking, and the field $\psi \propto\left(\boldsymbol{\Delta}_{1}^{2}+\boldsymbol{\Delta}_{2}^{2}+\boldsymbol{\Delta}_{3}^{2}\right)$ associated with the Gaussian fluctuations of the $\boldsymbol{\Delta}_{i}$ fields. Details of the Hubbard-Stratonovich transformation can be found in the Appendix. In particular, we show that the nonzero values of $\zeta_{1}$ and $\zeta_{2}$ are energetically unfavorable because $v>0$, so we set $\zeta_{1}=\zeta_{2}=0$ in the following analysis and consider states that preserve the lattice rotational symmetry.

The quartic terms in Eq. (5) can be decoupled using the auxiliary fields $\phi_{i}$ and $\psi$. Because we allow the $\boldsymbol{\Delta}_{i}$ fields to fluctuate, we include nonuniform space/time configurations, i.e., replace $\boldsymbol{\Delta} \rightarrow \boldsymbol{\Delta}_{q, \omega}$ and $r_{0} \rightarrow r_{0}+q^{2}+\Gamma\left|\omega_{m}\right|$ in Eq. (5), with $\omega_{m}=2 m \pi T$. Near a finite-temperature phase transition, thermal fluctuations are the most relevant, and we restrict our analysis to the $\omega_{m}=0$ component. The new action now depends only on the $\psi$ and $\boldsymbol{\phi}$ fields:

$$
S[\psi, \boldsymbol{\phi}]=\int_{x}\left(\frac{|\boldsymbol{\phi}|^{2}}{2 g}-\frac{\psi^{2}}{2 u}\right)+\frac{3}{2} \int_{q} \ln (\operatorname{det} \hat{\mathcal{X}}),
$$

where $|\boldsymbol{\phi}|^{2}=\sum_{i} \phi_{i}^{2}, \int_{q}=\frac{V T}{(2 \pi)^{d}} \int d^{d} q$, and $V$ is the volume of the system. The matrix $\hat{\mathcal{X}}$ is

$$
\hat{\mathcal{X}}=\left(\begin{array}{ccc}
\tilde{\chi}_{q}^{-1} & -\phi_{3} & -\phi_{2} \\
-\phi_{3} & \tilde{\chi}_{q}^{-1} & -\phi_{1} \\
-\phi_{2} & -\phi_{1} & \tilde{\chi}_{q}^{-1}
\end{array}\right),
$$

with renormalized $\tilde{\chi}_{q}^{-1}=r_{0}+\psi+q^{2} \equiv r+q^{2}$. In the absence of broken $Z_{4}$ symmetry, long-range SDW order sets in at $r=0$, hence, an intermediate phase exists if $Z_{4}$ symmetry is broken at some $r>0$.

The action (7) is an unconstrained function of $\psi$, which is the usual situation for Gaussian fluctuations, ${ }^{20}$ and reflects the fact that $\left\langle\boldsymbol{\Delta}_{i}^{2}\right\rangle \neq 0$. However, we are principally interested in the fields $\phi_{i}$, which have zero expectation value in the absence of $Z_{4}$ symmetry breaking. The mean-field theory for the action (7) is the set of coupled saddle-point equations, the minimum with respect to fluctuating fields $\phi_{i}$ and the maximum with respect to $\psi$.

\section{B. Mean-field theory}

The four possible realizations for the $Z_{4}$ symmetry breaking correspond to $\phi_{i}= \pm \phi$ subject to the constraint $\phi_{1} \phi_{2} \phi_{3}>0$. After substituting this in Eq. (6), integrating over $d^{2} q$, and absorbing the factor $T$ into the couplings, we obtain

$$
\begin{aligned}
\frac{S[r, \phi]}{3 V}= & -\frac{\left(r-r_{0}\right)^{2}}{6 u}+\frac{3 \phi^{2}}{6 g}+\frac{3 r}{8 \pi} \\
& +\frac{1}{8 \pi}\left[(r-2 \phi) \ln \frac{\Lambda^{2}}{r-2 \phi}+2(r+\phi) \ln \frac{\Lambda^{2}}{r+\phi}\right],
\end{aligned}
$$

where $\Lambda$ is the upper momentum cutoff, and $r_{0}$, which is proportional to the temperature, is the control parameter. The renormalized $r$ is proportional to the inverse magnetic correlation length $\xi^{-2}$. Differentiating Eq. (8) with respect to $r$ and $\phi$ yields the coupled saddle-point equations which determine their values at a given $r_{0}$ :

$$
\begin{gathered}
r=r_{0}+\frac{\bar{u}}{2} \ln \frac{\Lambda^{6}}{(r-2 \phi)(r+\phi)^{2}}, \\
\phi=\bar{g} \ln \left(\frac{r+\phi}{r-2 \phi}\right),
\end{gathered}
$$

where the rescaled parameters are $\bar{u}=3 u / 4 \pi$ and $\bar{g}=g / 4 \pi$. For $\phi=0$ (i.e., when $Z_{4}$ is unbroken), Eq. (9) takes the familiar form $r+3 \bar{u} /\left(2 \ln \Lambda^{2} / r\right)=r_{0}$ whose solution is positive for 

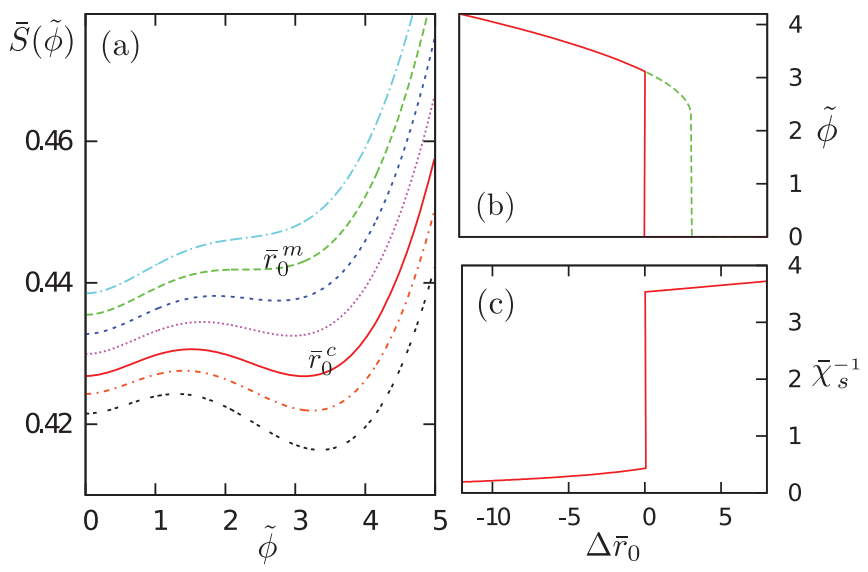

FIG. 4. (Color online) (a) The effective action $\bar{S}(\tilde{\phi})=S[r, \tilde{\phi}] / V \bar{g}$ as a function of $\tilde{\phi}$ for $\lambda=\bar{u} / \bar{g}=100$ and various $\bar{r}_{0}$. The different curves correspond to $\bar{r}_{0}=197,195.94\left(\bar{r}_{0}^{m}\right), 195,194,192.9\left(\bar{r}_{0}^{c}\right)$, 192, and 191 (from top to bottom). (b) The (red) solid curve shows the order parameter $\tilde{\phi}$ as a function $\Delta \bar{r}_{0}=\bar{r}_{0}-\bar{r}_{0}^{c}$. The (green) dashed curve shows the expectation value $\tilde{\phi}$ of the metastable phase for $\bar{r}_{0}<$ $\bar{r}_{0}^{m}$. (c) The inverse susceptibility of the singlet mode $1 / \chi_{s} \propto \bar{r}-2 \tilde{\phi}$ as a function of $\Delta \bar{r}_{0}$.

arbitrary $r_{0}$, i.e., $O(3)$ symmetry is preserved at any nonzero $T$. This is an obvious consequence of the Mermin-Wagner theorem. However, the discrete $Z_{4}$ symmetry can be broken at a finite $T$.

Assuming that $\phi$ is nonzero and eliminating $r$ from Eqs. (10) and (9), we obtain the self-consistent equation for the rescaled order parameter $\tilde{\phi}=\phi / \bar{g}$ :

$$
\tilde{\phi}\left(\frac{2 e^{\tilde{\phi}}+1}{e^{\tilde{\phi}}-1}\right)+\lambda\left[\tilde{\phi}+\frac{3}{2} \ln \left(\frac{3 \tilde{\phi}}{e^{\tilde{\phi}}-1}\right)\right]=\bar{r}_{0},
$$

where $\bar{r}_{0}=r_{0} / \bar{g}+(3 \bar{u} / 2 \bar{g}) \ln \left(\Lambda^{2} / \bar{g}\right) \propto\left(T-\bar{T}_{N}\right)$, and $\bar{T}_{N}$ is the rescaled mean field $T_{N}$. The ratio $\lambda \equiv \bar{u} / \bar{g}$ is large in our model, of order $W / T_{N}$, where $W$ is the bandwidth. ${ }^{10}$ The analysis of Eq. (11) for $\lambda \gg 1$ shows that the first nonzero solution appears at a particular temperature when $\bar{r}_{0}^{m} \approx$ $\frac{3}{2} \lambda \ln 3$ and at a finite $\tilde{\phi} \approx 2.15+14.2 / \lambda$. This obviously indicates that the mean-field $Z_{4}$ transition is first order. The actual transition temperature is smaller than $\bar{r}_{0}^{m}$ because at $\bar{r}_{0}^{m}$ the effective action only develops a local minimum at nonzero $\tilde{\phi}$, but this may not be a global minimum. To find when the actual transition occurs, we solve Eq. (9) for $r(\phi)$ numerically, substitute the result into (8), and obtain the effective action $S(\tilde{\phi})$ for which $\bar{r}_{0}$ is a parameter and Eq. (10) is the saddle-point solution. The behavior of $S(\tilde{\phi})$ for various $\bar{r}_{0}$ is shown in Fig. 4(a). At sufficiently large $\bar{r}_{0}$, it increases monotonically with $\tilde{\phi}$ and its only minimum is at $\tilde{\phi}=0$, implying that $Z_{4}$ is unbroken. At $\bar{r}_{0}=\bar{r}_{0}^{m}$, the function $S(\tilde{\phi})$ develops an inflection point, which at smaller $\bar{r}_{0}$ splits into a maximum and a minimum. At some $\bar{r}_{0}=\bar{r}_{0}^{c}$, the value of $S(\tilde{\phi})$ at this minimum becomes equal to $S(0)$, and for $\bar{r}_{0}<\bar{r}_{0}^{c}$, the global minimum of the free energy jumps to a finite $\tilde{\phi} \neq 0$. Once this happens, the system spontaneously chooses one out of four states with $\pm \tilde{\phi}$, and the $Z_{4}$ symmetry breaks down. We plot $\tilde{\phi}$ versus $\bar{r}_{0}$ in Fig. 4(b).
To find how much the $Z_{4}$ transition temperature $T_{Z_{4}}$ actually differs from the SDW transition temperature $\bar{T}_{N}$, we computed the spin susceptibility $\chi(q)$ within the random phase approximation (RPA), explicitly related $\bar{r}_{0}$ to $\left(T-\bar{T}_{N}\right)$, and expressed $\lambda$ in terms of the ratio of $T_{N}$ and the fermionic bandwidth $W$. Collecting all factors, we find

$$
T_{Z_{4}}=\bar{T}_{N}+a \frac{T_{N}^{2}}{W} \frac{1}{\ln W / T_{N}},
$$

where $a=O(1)$, and $T_{N}$ is the "mean-field" Neel temperature, which does not take into account the suppression of SDW order by thermal fluctuations. The actual $\bar{T}_{N}$ tends to zero in $2 \mathrm{D}$, but $T_{Z_{4}}$ remains finite.

To analyze how the broken $Z_{4}$ symmetry affects SDW correlations, we compute the eigenvalues of the spin susceptibility matrix $\hat{\mathcal{X}}$ in Eq. (7). The two eigenvalues correspond to a singlet and a doublet mode $\chi_{s}=1 /(r-2 \phi)$ and $\chi_{d}=1 /(r+\phi)$. If either $r-2 \phi$ or $r+\phi$ jumped to a negative value at the $Z_{4}$ transition, then the breaking of $Z_{4}$ would induce a simultaneous breaking of the $O(3)$ symmetry. However, it follows from (10) that both $\chi_{s}$ and $\chi_{d}$ remain finite when $\phi$ jumps to a nonzero value, i.e., breaking the $Z_{4}$ symmetry does not induce SDW order immediately [see Fig. 4(c)].

\section{Beyond mean field: 4-state Potts model}

In general, transitions into a symmetry-breaking phase with fourfold degeneracy fall into either the 4-state Potts or 4-state clock model. Our case belongs to the 4-state Potts universality class. This can be understood in several ways, e.g., by looking at the real-space picture of the $Z_{4}$ symmetry breaking. As shown in Fig. 1, there are four states corresponding to four possible choices where to place the center of the quadrupled unit cell. We labeled these four possibilities as A, B, C, and D. The geometrical equivalence of the four states is crucial here as the presence of four equivalent minima is the signature feature of the 4-state Potts model, while the clock model would correspond to, e.g., A being closer to B and D than to C, which is not the case here. This distinction is important, as they have different critical behaviors in two dimensions. While the 4-state Potts model has its own critical behavior, as discussed below, the 4-state clock model belongs to the universality class of the two-dimensional (2D) Ising model.

Another way to see the equivalence of our model to 4-state Potts model is to expand the effective action (8) for small $\phi$ and large $\lambda$ as

$$
\frac{S(\tilde{\phi})}{V}=\left(\bar{r}_{0}-\bar{r}_{0}^{m}\right) \tilde{\phi}^{2}-\frac{\lambda}{12} \tilde{\phi}^{3}+\frac{\lambda}{16} \tilde{\phi}^{4}+\cdots .
$$

The action of the 4-state Potts model has the same form, ${ }^{21}$ hence, both transitions belong to the same universality class. The cubic term in the action originates from the triple product $\phi_{1} \phi_{2} \phi_{3}$ in the expansion of the original mean-field action (6). Such a cubic term is consistent with the symmetry of the $Z_{4}$ order parameters.

To see this, we consider the transformation of the $Z_{4}$ order parameters under basic symmetry operations of the system. Obviously, $\phi_{i} \propto \boldsymbol{\Delta}_{j} \cdot \boldsymbol{\Delta}_{k}$ are invariant under spin rotations and particularly time-reversal transformation. Lattice reflections 
and rotations simply permute the three $\phi$ parameters, hence leaving the cubic term unchanged. Finally, since each $\phi_{i}$ carries the momentum $\mathbf{Q}_{i}$, these parameters acquire a phase $\exp \left(i \mathbf{Q}_{i}\right.$. $\mathbf{t})= \pm 1$ under lattice translation $\mathbf{t}$. It can be easily checked that for primitive lattice vectors $\mathbf{t}=(1,0)$ and $(1 / 2, \sqrt{3} / 2)$, only two of the three $\phi_{i}$ fields change sign, again leaving the triple product unchanged. These considerations show that the cubic term $\phi_{1} \phi_{2} \phi_{3}$ is an invariant of the symmetry group and is allowed in the Landau expansion.

The equivalence of our model to the Potts model allows us to go beyond the saddle-point solution and understand how the $Z_{4}$ transition is affected by fluctuations of $\phi$ fields. The 4-state Potts model in 2D does exhibit a transition, i.e., the preemptive $Z_{4}$ ordering is not destroyed by fluctuations. ${ }^{22}$ Interestingly, however, fluctuations transform the first-order transition into a second-order transition, although with a rather small critical exponent $\beta=1 / 12$ for $\phi \sim\left(T_{c}-T\right)^{\beta}$ (Ref. 22). A small $\beta$ implies that the order parameter sharply increases below the critical temperature, and in practice this behavior is almost indistinguishable from that in the first-order transition.

\section{Experimental manifestations}

As spin rotational symmetry is preserved in the preemptive $Z_{4}$ phase, no magnetic Bragg peaks are to be observed in neutron scattering experiments. On the other hand, since the charge density $\rho(\mathbf{r})$ and the Casimir operator $\mathbf{S}^{2}(\mathbf{r})$ have the same symmetry, a spatial modulation of the latter induces a modulation in the charge density. Given the 2D character of graphene, such a superlattice structure can be directly probed by STM. The additional Bragg peaks due to the quadrupled unit cell should also be detectable by scattering measurements. Local probes such as NMR can measure the different onsite fluctuating magnetic moments of the $Z_{4}$ phase since the size of the local moment controls the linewidth of the NMR signal. We thus expect to see two different linewidths coming from the $3 \Delta$ and the $\Delta$ sites.

The order parameter $\phi$ can also be inferred by measuring the static magnetic susceptibility $\chi$ at any of the three nesting vectors. In the absence of $O(3)$ breaking, we have $\chi\left(\bar{r}_{0}\right)=\left(2 \chi_{d}+\chi_{s}\right) / 3$. Once the order parameter $\phi$ jumps to a finite value below the transition, so does the susceptibility $\chi\left(\bar{r}_{0}\right)=\tilde{r}^{-1}+\phi^{2} \tilde{r}^{-3}+\cdots$, where $\tilde{r}$ is the value of $r$ at $\phi=0$. This provides a direct method for detecting the order parameter $\phi$. The jump of the static susceptibility (i.e., of the spin correlation length) also affects the electronic spectrum. For larger correlation length, the system develops precursors to the SDW order, which give rise to a pseudogap in the electronic spectral function. This pseudogap can be probed by photoemission experiments. ${ }^{6}$

\section{CONCLUSION AND DISCUSSION}

We discussed in this work the intriguing possibility of an emergent paramagnetic phase with spontaneously broken translational symmetry for properly doped fermions on triangular and hexagonal lattices. This unique state emerges from a preemptive phase transition which breaks only a discrete translational $Z_{4}$ lattice symmetry but preserves $O(3)$ spinrotational invariance. We demonstrated that this phase exists in $2 \mathrm{D}$ systems and by continuity should exist in anisotropic 3D systems. We argued that such a phase should be observed in STM, NMR, neutron scattering, and photoemission experiments.

Because each $\phi$ is constructed out of a product of two $\Delta$ 's, the $Z_{4}$ order shown in Fig. 2 does not directly couple to fermions and does not give rise to a sharp gap in the fermionic dispersion, hence the system remains a metal. At the same time, $Z_{4}$ order almost discontinuously increases magnetic correlation length and by virtue of this gives rise to magnetic precursors which tend to partly shift a spectral weight from low energies to a finite frequency, as in a SDW-ordered state.

One particular interesting issue left for further studies is how the $Z_{4}$-ordered state is affected by impurities. In general, impurities should be pair breaking for all ordered states: $d+i d$ superconductor, SDW state, and $Z_{4}$ state, but how they affect each of these states requires a separate study.

\section{ACKNOWLEDGMENTS}

We acknowledge useful conversations with L. Levitov, P. Lee, L. Fu, C. D. Batista, I. Martin, and P. Kim. G.W.C. is supported by ICAM and Grant No. NSF-DMR-0844115, R.M.F. acknowledges the support from ICAM and Grant No. NSF-DMR 0645461, as well as the valuable support from the NSF Partnerships for International Research and Education (PIRE) Program No. OISE-0968226. A.V.C. is supported by NSF-DMR-0906953.

\section{APPENDIX: HUBBARD-STRATONOVICH TRANSFORMATION}

In this Appendix, we present the details of the HubbardStratonovich transformation for the preemptive phase. We first introduce six bosonic fields $\psi, \zeta_{1}, \zeta_{2}$, and $\phi_{i}(i=1,2,3)$, each corresponding to one of the fourth-order terms in Eq. (5) of the main text. Explicitly, the interaction terms in the partition function $Z=\int \mathcal{D} \boldsymbol{\Delta}_{i} \exp \left(-S\left[\boldsymbol{\Delta}_{i}\right]\right)$ can be rewritten as

$$
\begin{aligned}
& \exp \left[-\frac{u}{2} \int_{x}\left(\boldsymbol{\Delta}_{1}^{2}+\boldsymbol{\Delta}_{2}^{2}+\boldsymbol{\Delta}_{3}^{2}\right)^{2}\right]=\int \mathcal{D} \psi \exp \int_{x}\left[\frac{\psi^{2}}{2 u}-\psi\left(\boldsymbol{\Delta}_{1}^{2}+\boldsymbol{\Delta}_{2}^{2}+\boldsymbol{\Delta}_{3}^{2}\right)\right], \\
& \exp \left[-\frac{v}{2} \int_{x}\left(\boldsymbol{\Delta}_{1}^{2}+\boldsymbol{\Delta}_{2}^{2}-2 \boldsymbol{\Delta}_{3}^{2}\right)^{2}\right]=\int \mathcal{D} \zeta_{1} \exp \int_{x}\left[\frac{\zeta_{1}^{2}}{2 v}-\zeta_{1}\left(\boldsymbol{\Delta}_{1}^{2}+\boldsymbol{\Delta}_{2}^{2}-2 \boldsymbol{\Delta}_{3}^{2}\right)\right],
\end{aligned}
$$




$$
\begin{gathered}
\exp \left[-\frac{v}{2} \int_{x} 3\left(\boldsymbol{\Delta}_{1}^{2}-\boldsymbol{\Delta}_{2}^{2}\right)^{2}\right]=\int \mathcal{D} \zeta_{2} \exp \int_{x}\left[\frac{\zeta_{2}^{2}}{2 v}-\sqrt{3} \zeta_{2}\left(\boldsymbol{\Delta}_{1}^{2}-\boldsymbol{\Delta}_{2}^{2}\right)\right], \\
\exp \left[\frac{g}{2} \int_{x}\left(\boldsymbol{\Delta}_{i} \cdot \boldsymbol{\Delta}_{j}\right)^{2}\right]=\int \mathcal{D} \phi_{k} \exp \int_{x}\left[-\frac{\phi_{k}^{2}}{2 g}+\phi_{k}\left(\boldsymbol{\Delta}_{i} \cdot \boldsymbol{\Delta}_{j}\right)\right],
\end{gathered}
$$

where $(i j k)$ in the last equation are cyclic permutations of (123). The new action becomes

$$
S\left[\boldsymbol{\Delta}_{i}, \psi, \zeta, \boldsymbol{\phi}\right]=\sum_{i j} \int_{q} \mathcal{X}_{i j}[\psi, \zeta, \boldsymbol{\phi}]\left(\boldsymbol{\Delta}_{i} \cdot \boldsymbol{\Delta}_{j}\right)+\int_{x}\left(\frac{|\boldsymbol{\phi}|^{2}}{2 g}-\frac{|\zeta|^{2}}{2 v}-\frac{\psi^{2}}{2 u}\right),
$$

where $\zeta=\left(\zeta_{1}, \zeta_{2}\right)$ and $\boldsymbol{\phi}=\left(\phi_{1}, \phi_{2}, \phi_{3}\right)$, and the matrix $\hat{\mathcal{X}}$ is

$$
\hat{\mathcal{X}}=\left(\begin{array}{lcc}
\tilde{\chi}_{q}^{-1}+2 \hat{\mathbf{u}}_{1} \cdot \zeta & -\phi_{3} & -\phi_{2} \\
-\phi_{3} & \tilde{\chi}_{q}^{-1}+2 \hat{\mathbf{u}}_{2} \cdot \zeta & -\phi_{1} \\
-\phi_{2} & -\phi_{1} & \tilde{\chi}_{q}^{-1}+2 \hat{\mathbf{u}}_{3} \cdot \zeta
\end{array}\right),
$$

with $\tilde{\chi}_{q}^{-1}=r_{0}+\psi+q^{2} \equiv r+q^{2}$, and the three unit vectors are $\hat{\mathbf{u}}_{1,2}=(1 / 2, \pm \sqrt{3} / 2)$ and $\hat{\mathbf{u}}_{3}=(-1,0)$. Integrating out the $\boldsymbol{\Delta}_{i}$ fields yields an effective action

$$
\begin{aligned}
S[\psi, \zeta, \boldsymbol{\phi}]= & \frac{3}{2} \int_{q} \ln (\operatorname{det} \hat{\mathcal{X}}[\psi, \zeta, \boldsymbol{\phi}]) \\
& +\int_{x}\left(\frac{|\boldsymbol{\phi}|^{2}}{2 g}-\frac{|\zeta|^{2}}{2 v}-\frac{\psi^{2}}{2 u}\right) .
\end{aligned}
$$

As discussed in the main text, the mean-field solution of the potential preemptive phase is given by the saddle-point solution of coupled equations: $\partial S / \partial \psi=\partial S / \partial \zeta=\partial S / \partial \boldsymbol{\phi}=$ 0 . In particular, we consider the two equations involving the doublet $\zeta$ :

$$
\begin{aligned}
& \zeta_{1}=\frac{3 v}{2} \int_{q} \frac{6 \zeta_{2}^{2}-6 \zeta_{1}\left(\tilde{\chi}_{q}^{-1}+\zeta_{1}\right)-\left(\phi_{1}^{2}+\phi_{2}^{2}-2 \phi_{3}^{2}\right)}{\operatorname{det} \hat{\mathcal{X}}}, \\
& \zeta_{2}=\frac{3 v}{2} \int_{q} \frac{6 \zeta_{2}\left(2 \zeta_{1}-\tilde{\chi}_{q}^{-1}\right)-\sqrt{3}\left(\phi_{1}^{2}-\phi_{2}^{2}\right)}{\operatorname{det} \hat{\mathcal{X}}} .
\end{aligned}
$$

It can be easily checked that the mean-field configurations with $\zeta_{1}=\zeta_{2}=0$ and $\left|\phi_{1}\right|=\left|\phi_{2}\right|=\left|\phi_{3}\right|=\phi$ are solutions of the above two equations, indicating that the $Z_{4}$ phase solutions discussed in the main text satisfy the saddle-point equations of the effective action (A7).
${ }^{1}$ P. Chandra, P. Coleman, and A. I. Larkin, Phys. Rev. Lett. 64, 88 (1990).

${ }^{2}$ J. T. Chalker, P. C. W. Holdsworth, and E. F. Shender, Phys. Rev. Lett. 68, 855 (1992).

${ }^{3}$ G. Möller and R. Moessner, Phys. Rev. B 80, 140409(R) (2009); G.-W. Chern, P. Mellado, and O. Tchernyshyov, Phys. Rev. Lett. 106, 207202 (2011).

${ }^{4}$ R. A. Borzi, S. A. Grigera, J. Farrell, R. S. Perry, S. J. S. Lister, S. L. Lee, D. A. Tennant, Y. Maeno, and A. P. Mackenzie, Science 315, 214 (2007).

${ }^{5}$ J.-H. Chu, J. G. Analytis, K. De Greve, P. L. McMahon, Z. Islam, Y. Yamamoto, and I. R. Fisher, Science 329, 824 (2010).

${ }^{6}$ R. M. Fernandes, A. V. Chubukov, J. Knolle, I. Eremin, and J. Schmalian, Phys. Rev. B 85, 024534 (2012) and references therein. ${ }^{7}$ I. Eremin and A. V. Chubukov, Phys. Rev. B 81, 024511 (2010).

${ }^{8}$ A. H. Castro Neto, F. Guinea, N. M. R. Peres, K. S. Novoselov, and A. K. Geim, Rev. Mod. Phys. 81, 109 (2009).

${ }^{9}$ J. L. McChesney, A. Bostwick, T. Ohta, T. Seyller, K. Horn, J. Gonzalez, and E. Rotenberg, Phys. Rev. Lett. 104, 136803 (2010).

${ }^{10}$ R. Nandkishore, G.-W. Chern, and A. V. Chubukov, Phys. Rev. Lett. 108, 227204 (2012).
${ }^{11}$ R. Nandkishore, L. Levitov, and A. Chubukov, Nat. Phys. 8, 158 (2012).

${ }^{12} \mathrm{~T}$. Li, arXiv:1103.2420.

${ }^{13}$ D. Makogon, R. van Gelderen, R. Roldan and C. Morais Smith, Phys. Rev. B 84, 125404 (2011).

${ }^{14}$ A. Smolyanitsky, J. P. Killgore, and V. K. Tewary, Phys. Rev. B 85, 035412 (2012)

${ }^{15}$ B. Valenzuela and M. A. H. Vozmediano, New J. Phys. 10, 113009 (2008).

${ }^{16}$ M. Kiesel, C. Platt, W. Hanke, D. A. Abanin, and R. Thomale, arXiv:1109.2953.

${ }^{17}$ J. Gonzalez, Phys. Rev. B 78, 205431 (2008).

${ }^{18}$ T. Li, arXiv:1001.0620.

${ }^{19}$ I. Martin and C. D. Batista, Phys. Rev. Lett. 101, 156402 (2008).

${ }^{20} \mathrm{P}$. M. Chaikin and T. C. Lubensky, Principles of Condensed Matter Physics (Cambridge University Press, Cambridge, UK, 1995).

${ }^{21}$ R. K. P. Zia and D. J. Wallace, J. Phys. A: Math. Gen. 8, 1495 (1975).

${ }^{22}$ F. Y. Wu, Rev. Mod. Phys. 54, 235 (1982). 